ORIGINAL ARTICLE/ARTIGO ORIGINAL

\title{
Association between dental visits at primary care and glycated hemoglobin level in patients with type 2 diabetes: a cohort study
}

\author{
Associação entre visitas odontológicas na a tenção primária e níveis de \\ hemoglobina glicada em pacientes com diabetes tipo 2: estudo de coorte
}

\author{
Aurea Letícia Horbach' (D), Julio Baldisserotto!,I (D), Roger Keller Celeste" (i)
}

\begin{abstract}
Objective: To evaluate the association between dental visits and variation in the glycated hemoglobin index (A1C) of patients with type 2 diabetes (T2DM) with well or not well glycemic control over time. Methods: Patients with T2DM, A1C $\geq 7 \%$ (not well-controlled) and $<7 \%$ (well-controlled), who attended a primary care service and were followed up from January 2010 to May 2018. The outcome was the variation of A1C obtained from reference laboratories. At the beginning of the study, a questionnaire with behavioral, clinical, and socioeconomic information was carried out. Multiple linear regression analyses tested interaction terms of all variables with the initial glycemic level (not well-controlled or well-controlled). Results: The sample consisted of 507 people, $65 \%$ women, and $66 \%$ individuals 55 to 74 years old, followed on average for 5.4 years. There was an interaction $(\mathrm{p}=0.01)$ between dental visits and initial A1C. Patients not well-controlled with at least one dental visit had an average reduction in $\mathrm{A} 1 \mathrm{C}$ of -0.56 percentage point $(95 \% \mathrm{CI}$ -1.06- -0.56), whereas the well-controlled group who also had at least one dental visit had an increase of 0.34 percentage point (95\%CI -0.18 - 0.87). Conclusion: Dental visits were associated with an improvement in A1C of approximately a half-percentage point in patients who had the initial A1C considered as not well-controlled.
\end{abstract}

Keywords: Diabetes mellitus. Primary health care. Oral health. Dental care.

'Conceição Hospital Group - Porto Alegre (RS), Brazil.

"Department of Preventive and Social Dentistry, Universidade Federal do Rio Grande do Sul - Porto Alegre (RS), Brazil.

Corresponding author: Roger Keller Celeste. Department of Preventive and Social Dentistry, Faculty of Dentistry, Universidade Federal do Rio Grande do Sul. Rua Ramiro Barcelos, 2.492, 30 andar, CEP: 90035-003, Porto Alegre, RS, Brazil. E-mail: roger.keller@ufrgs.br Conflict of interests: nothing to declare - Financial support: National Council for Scientific and Technological Development (CNPq). 
RESUMO: Objetivo: Avaliar a associação entre consultas odontológicas e variação no índice de hemoglobina glicada (A1C) em pacientes com diabetes mellitus tipo 2 (DM2) com controle glicêmico bom ou não ao longo do tempo. Métodos: Pacientes com DM2, A1C $\geq 7 \%$ (não controlado) e $<7 \%$ (bem controlado), que compareceram a um serviço de atenção primária e foram acompanhados de janeiro de 2010 a maio de 2018 . O desfecho foi a variação de A1C, obtidos em laboratórios de referência. No início do estudo, foi aplicado um questionário com informações comportamentais, clínicas e socioeconômicas. Foram ajustados modelos de regressão linear múltipla para controle de fatores de confusão, e testou-se a interação de todas as variáveis com o nível glicêmico inicial (não controlado ou controlado). Resultados: A amostra foi composta de 507 pessoas, $65 \%$ mulheres e $66 \%$ indivíduos de 55 a 74 anos, acompanhados em média por 5,4 anos. Houve interação $(\mathrm{p}=0,01)$ entre as consultas odontológicas e níveis iniciais de A1C. Pacientes não controlados com pelo menos uma consulta odontológica tiveram redução média de A1C de $-0,56$ pontos percentuais (intervalo de confiança de $95 \%$ - IC $95 \%-1,06--0,56$ ), enquanto o grupo controlado que também teve pelo menos uma consulta odontológica teve aumento de 0,34 ponto percentual (IC $95 \%-0,18$ - 0,87). Conclusão: As visitas ao dentista foram associadas à melhora na $\mathrm{A} 1 \mathrm{C}$ de aproximadamente meio ponto percentual em pacientes que tiveram a $\mathrm{A} 1 \mathrm{C}$ inicial considerada não bem controlada.

Palavras-chave: Diabetes mellitus. Atenção primária à saúde. Saúde bucal. Assistência odontológica.

\section{INTRODUCTION}

The global prevalence of Diabetes Mellitus (DM) has greatly increased over the last decades, especially in low and middle-income countries ${ }^{1}$. Both the disease and its complications or comorbidities are important risk factors, causing deaths worldwide ${ }^{2}$. According to the Global Disease Burden Study, the participation of Type 2 Diabetes Mellitus (T2DM) was the most significant among the chronic non-communicable diseases in the Brazilian epidemiological scenario in 2008, ranking among the top five disorders of the disease burden in the country ${ }^{3}$. Considering the current and growing load involved, actions to control and prevent DM are an urgent public health issue $e^{4}$.

The treatment of a patient with T2DM aims to maintain adequate blood glucose levels since chronic hyperglycemia is associated with micro and macrovascular complications ${ }^{5}$. Several oral diseases have also been reported in these patients, as greater severity and prevalence of periodontitis ${ }^{6.8}$, more tooth $\operatorname{loss}^{6,9}$, higher caries index ${ }^{10,11}$, increased prevalence of candidiasis ${ }^{12}$, xerostomia ${ }^{12}$, and oral cancer ${ }^{6}$.

In particular, the association between periodontal disease and DM has been extensively explored over the years, and it is the only one among these conditions considered with a bidirectional effect in the control of glycemia ${ }^{13}$. On the one hand, there is increased prevalence and severity of periodontal disease among patients with diabetes ${ }^{14}$ and, on the other, there is the possibility of the periodontal disease to raise systemic inflammatory burden and contribute to increased risk of complications ${ }^{15}$. However, few studies in the literature about the influence of access to dental care in a primary health care scenario have been found. 
Controlling the common risk factors for many chronic non-communicable diseases can be a shared role for oral health professionals ${ }^{16}$. Oral health is also determined by diet, hygiene, smoking, alcohol use, stress, and trauma, and a collaborative approach related to these factors can promote better health status with reduced cost, increased effectiveness, and efficiency than adopting isolated approaches ${ }^{17}$. Considering Primary Health Care as an essential setting to promote the health of patients with T2DM, in which oral care is included, the present study aims to evaluate the association between dental visits and variation in the glycated hemoglobin index (A1C) of patients with type 2 diabetes (T2DM) with well or notwell glycemic control over time.

\section{METHODS}

\section{STUDY POPULATION AND DATA COLLECTION}

A fixed cohort study was performed in adult patients with T2DM residents in the area covered by the Community Health Service of the Conceição Hospital Group (CHS / CHG). The CHS/ CHG is a public service integrated with the Brazilian Unified Health System (SUS) and based on Primary Health Care principles in Porto Alegre, a city in Brazil's southern region. From the population enrolled in the CHS / CHG system $(\sim 108$ thousand people), it was estimated that $8 \%$ had T2DM. The Research Ethics Committee approved the study protocol of Nossa Senhora da Conceição Hospital (Protocol No. 10261). The written Informed Consent Form was obtained from all participants included in the study.

The cohort's time frame started in 2011; when structured interviews were conducted at the participants' houses, the study was called "Evaluation of Health Care in Hypertension and Diabetes in Primary Care". The baseline sample was randomly selected from those enrolled in the CHS / CHG health information system. To participate in research cited, individuals had to meet the following inclusion criteria:

- having a confirmed diagnosis of hypertension or diabetes (using clinical and laboratory standard tests) in the CHS/CHG Service Information System that included clinical and laboratory exams in 2009-2011 (before the interviews);

- having consulted in the health system at least once in the three years before the study recruitment;

- being 18 or older by March $1^{\text {st }}, 2009$.

Out of 9,059 eligible patients (baseline), a randomized selection of 3,784 individuals was performed, of which 2,482 were effectively enrolled $(65.6 \% \text { response rate })^{18}$. The exclusions occurred due to individuals not being found after four attempts, refusal to participate, change of address, or death. The selection process is illustrated in Figure 1.

Socioeconomic, demographic, behavioral, and lifestyle data were obtained retrospectively in 2011 from structured interviews conducted at the participants' houses by a company 
Figure 1 Flowchart of sample selection at baseline (2009-2011) and last follow-up (2018)

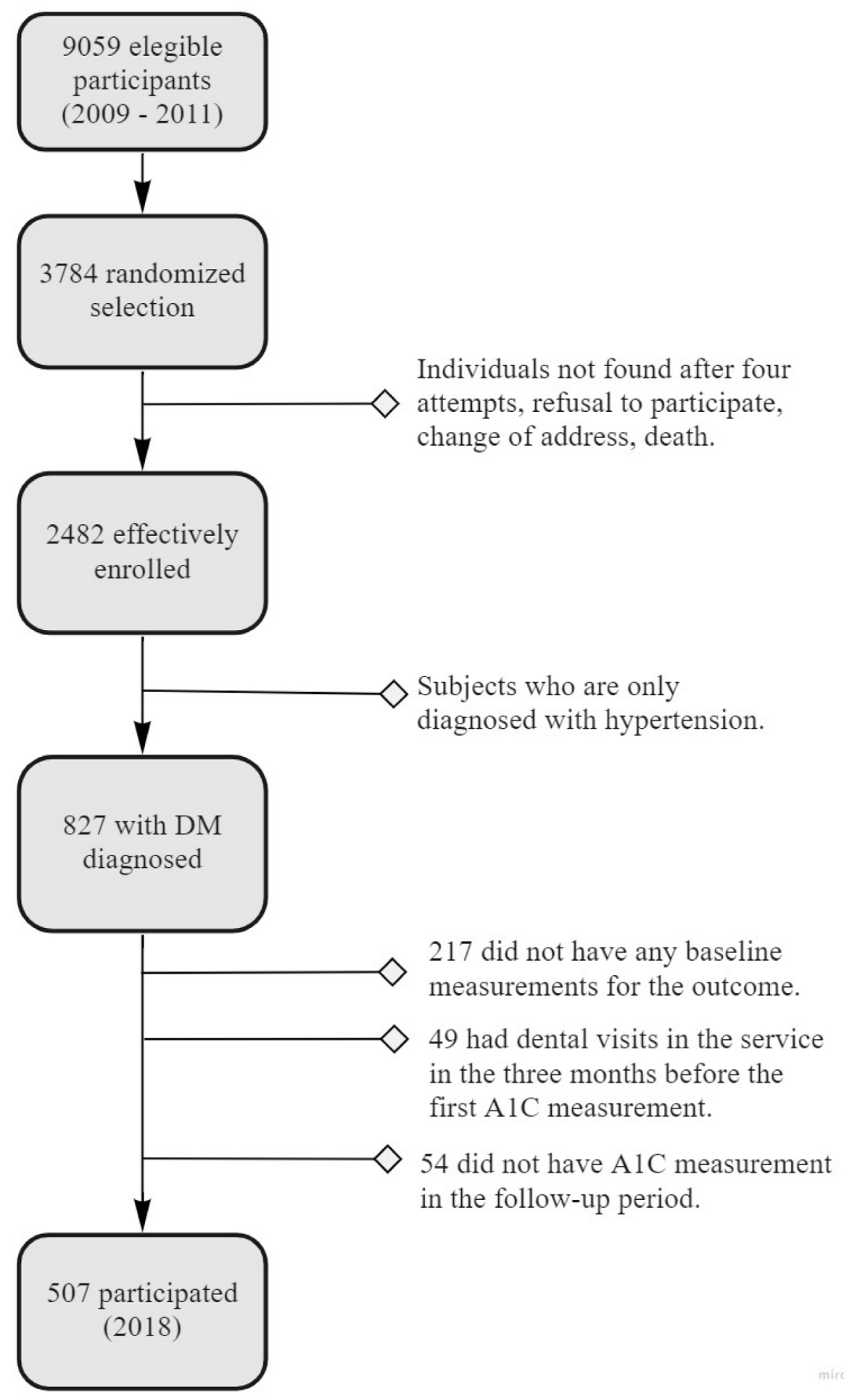


specialized in population studies. Trained interviewers conducted data collection using questionnaires only; they were not health professionals ${ }^{18}$. Baseline clinical data were obtained from existing information in the CHS / CHG health information system between 2010-2012. Therefore, the first measurement of A1C dates from 2010-2012. The final A1C measurement dates from 2012-2018.

For present research, 1,655 subjects who were only diagnosed with hypertension without diabetes were excluded. Besides them, other 217 were excluded because they did not have any baseline measurements for the outcome. Since dental visits were the primary variable of interest, 49 additional people were also excluded because they had dental visits in the service in the three months before the first A1C measurement, and this visit could affect the patient's average blood glucose levels and the first A1C measurement. From the 561 patients that started, 54 were lost because they did not have A1C measurement in the follow-up period between 2013 and 2018 (9.6\% attrition rate).

The sample was divided into two groups, according to the initial glycemic control. Participants with the initial A1C lower than $7 \%$ were considered the well-controlled group, and those equal to or greater than $7 \%$ were the not well-controlled group. Glycemic control goal for most patients with T2DM is known for maintaining the A1C measurement below $7 \%$; a more or less rigid target may be indicated depending on individual characteristics, such as the presence of comorbidities and type of treatment adopted.

\section{OUTCOME VARIABLE AND MAIN EXPOSURE}

The primary exposure was dental visits for any reason (dental check-up, oral diagnosis, urgent care, prevention, treatment) in the CHS / CHG during the period established as a follow-up. This variable was obtained from the CHS / CHG health information system and categorized into "no dental visit" during follow-up or "some/ several" because the distribution was skewed, and few individuals had more than one visit.

The outcome was the change in glycemic control from baseline to the final measurement (incidence measure) in the system regarding the hemoglobin A1C test measured in percentage points (pp). Such data were obtained in the CHS/CHG health information system, an electronic medical record maintained by health professionals during the follow-up of these patients in the service. A1C change was used as a continuous scale.

\section{COVARIATES}

The group of demographic factors included three variables. Gender was defined as male or female. Participants were divided into three age groups: up to 54 years old, between 55 and 74 years old, and equal to or over 75 years old. Marital status was defined as "living with a partner" for individuals who responded that they were married or lived 
with a partner, and "living alone" for the respondents who were single, widowed, separated, or divorced.

Socioeconomic factors comprised two variables. The classification criteria of the Brazilian Association of Market Research Companies (Associação Brasileira de Empresas de Pesquisas - ABEP) was used to define the economic situation of participants using the purchasing power and level of education of the head of the household, ranging from the highest to the lowest level, in the A/B, C, and D/E classes. Schooling was measured at educational attainment and dichotomized as $<$ elementary or $\geq$ elementary school, representing four years of study.

Behavioral and lifestyle factors included six variables. To define adequate or inadequate diet, interviewers asked questions proposed by the Brazilian Food Guide for the Brazilian population about the consumption of fruits, vegetables, fish, fried foods, sausages, sweets, soft drinks, besides the habit of removing visible fat from meats. Diet's adequacy was classified into three levels, according to the points obtained in the questionnaire. These data came from a previous study ${ }^{19}$, and for analysis purposes in this work, the category "partially adequate diet" was grouped into the "appropriate diet category". Tow measure of a sedentary lifestyle (yes or no) was obtained, one at baseline and the other closest measure to $\mathrm{A} 1 \mathrm{C}$, according to information filled out by doctors or nurses. According to data in the Information System database, patients were not considered sedentary when performing at least 150 minutes of light or moderate physical activity per week. Smoking was collected in three categories: former smoker, current smoker, or never smoker. DM medication use and attendance at diabetic/hypertensive groups were self-reported and categorized as yes or no. Finally, the number of specific doctor visits for this condition was defined as medical appointments for diabetes and collected from the CHS / CHG health information system.

Two variables on health status were used. Body mass index (BMI) was calculated through the answers obtained on weight and height and interpreted according to the World Health Organization in underweight, normal weight, overweight, and obesity ${ }^{20}$. The number of teeth was obtained from the self-report and dichotomized in the median $(<10$ teeth or $\geq 10$ teeth).

\section{STATISTICAL ANALYSIS}

First, bivariate analyses were performed with the non-parametric Mann-Whitney or Kruskal-Wallis test. Multiple linear regression analysis was carried out by testing interaction terms of all variables with the initial glycemic level (well-controlled versus not well-controlled). The final model was adjusted using a backward stepwise technique with $\mathrm{p}<0.10$ if there was a significant interaction at a 5\% level. The residuals of the final model were evaluated concerning normality, heteroskedasticity, variance inflation factor, and the presence of outliers with leverage power. For presentation, a stratified table was made with mutually adjusted coefficients. All analysis was performed using the Stata software, version 13.1. 


\section{RESULTS}

In total, 507 people participated. They were followed for a mean of 5.4 years (maximum of 8.33 and a minimum of 0.45 years). At baseline, 269 patients had initial A1C equal to or greater than $7 \%$ and were allocated in the not well-controlled group. They were followed for 5.4 years on average. The remaining 238 individuals had an initial A1C measurement lower than $7 \%$, were part of the well-controlled group, and were followed for 5.3 years on average. The follow-up period length was not statistically significant related to A1C variation $(\mathrm{p}=0.81)$. Table 1 shows the means and the medians of the follow-up time between the first and last $\mathrm{A} 1 \mathrm{C}$ measurements.

The descriptive characteristics of participants and bivariate associations between the A1C variation with sociodemographic and behavioral characteristics were shown in Tables 2 and 3. The sample consisted mainly of women ( $65 \%$ of participants) and individuals between 55 and 74 years old $(66 \%)$. Of the total sample, $55 \%$ of the individuals reported living with their partners, and $57 \%$ of participants belonged to Economic Class C. At baseline, $72 \%$ completed elementary education or more, $41 \%$ of the individuals were overweight, and $34 \%$ were obese. Only $12 \%$ were current smokers, whereas nearly half had never smoked. The diet was considered adequate for $67 \%$ of individuals. A sedentary lifestyle was measured with longitudinal data, and $69 \%$ of participants started the study and remained sedentary until the last measurement of their last glycemia result. During follow-up, $35 \%$ of the total sample did not visit the dentist in the CHS / CHG service. Of those with A1C values of not well-control at baseline, $36 \%$ did not visit the Dentist, whereas $34 \%$ in the well-controlled group did not visit the dentist.

The overall mean change in A1C was $-0.02 \mathrm{pp}( \pm \mathrm{SD} 1.95, \mathrm{~min}=-8.2$, median $=0$, $\max$ $=8.5$ ) in all groups. Among those who initially did not have well-controlled A1C values, the mean reduction was $-0.59 \mathrm{pp}( \pm \mathrm{SD} 2.16)$. Among those in the well-controlled A1C group, it increased by $0.63 \mathrm{pp}( \pm$ SD 1.43$)$ on average in the period.

Table 1 Follow-up time in years between the first and last A1C measurement

\begin{tabular}{l|c|c|c|}
\hline & Total sample & Initial A1C $\geq 7 \%$ & Initial A1C $<7 \%$ \\
\hline Mean (years) & 5.4 & 5.4 & 5.3 \\
\hline $95 \% \mathrm{Cl}$ & $(5.2-5.5)$ & $(5-5.5)$ & $(5.2-5.7)$ \\
\hline Minimum/Maximum (years) & $0.45 / 8.33$ & $0.45 / 8.33$ & $0.53 / 7.99$ \\
\hline $25^{\text {th }}$ percentile & 4.1 & 3.9 & 4.2 \\
\hline Median & 6.2 & 6.1 & 6.2 \\
\hline $75^{\text {th }}$ percentile & 6.8 & 6.9 & 6.8
\end{tabular}

A1C: glycated hemoglobin index; 95\%Cl: 95\% confidence interval. 
Table 2 Mean change of the glycated hemoglobin (A1C) in percentage points (pp) and standard deviation $( \pm S D)$ in patients of a Primary Health Care Service, according to the initial values and the association with covariables

\begin{tabular}{|c|c|c|c|c|c|}
\hline & $\mathrm{N}$ & Percentage & Change in $\mathrm{A} 1 \mathrm{C} p \mathrm{p}$ & $( \pm \mathrm{SD})$ & $p$-value \\
\hline Total & 507 & $100.0 \%$ & -0.02 & $(1.95)$ & \\
\hline \multicolumn{6}{|l|}{ Initial A1C } \\
\hline$\geq 7 \%$ & 269 & $53.1 \%$ & -0.59 & $(2.16)$ & $<0.01$ \\
\hline$<7 \%$ & 238 & $46.9 \%$ & 0.63 & $(1.43)$ & \\
\hline \multicolumn{6}{|l|}{ Gender } \\
\hline Male & 178 & $35.1 \%$ & 0.03 & $(1.88)$ & 0.32 \\
\hline Female & 329 & $64.9 \%$ & -0.05 & (1.98) & \\
\hline \multicolumn{6}{|l|}{ Age at baseline } \\
\hline$<55$ & 82 & $16.2 \%$ & 0.38 & $(2.49)$ & 0.04 \\
\hline $55-74$ & 332 & $65.5 \%$ & -0.06 & $(1.92)$ & \\
\hline$\geq 75$ & 93 & $18.3 \%$ & -0.23 & (1.37) & \\
\hline \multicolumn{6}{|l|}{ Diet } \\
\hline Adequate & 340 & $67.1 \%$ & -0.06 & (1.96) & 0.27 \\
\hline Inadequate & 106 & $20.9 \%$ & 0.28 & $(1.94)$ & \\
\hline \multicolumn{6}{|l|}{ Dental visits } \\
\hline None & 177 & $34.9 \%$ & 0.07 & $(1.63)$ & 0.94 \\
\hline Some/several & 330 & $65.1 \%$ & -0.07 & $(2.10)$ & \\
\hline \multicolumn{6}{|c|}{ Medical appointments for diabetes melitus } \\
\hline None & 185 & $36.5 \%$ & -0.01 & $(1.75)$ & 0.11 \\
\hline 1 to 2 times & 200 & $39.4 \%$ & -0.19 & $(2.06)$ & \\
\hline$>3$ times & 121 & $23.9 \%$ & 0.21 & $(2.01)$ & \\
\hline
\end{tabular}

A1C: glycated hemoglobin index.

Four variables were included in the final multiple linear regression model: dental visits, age, diet, and baseline glycemic control (Table 4), but due to missing in at least one variable, the final analytical sample was 446 individuals. Multiple regression showed that, at baseline, older people reduced the A1C annually on average $-0.02 \mathrm{pp}(95 \% \mathrm{CI}-0.04--0.01)$ by controlling their diet, glycemic control, and dental visits. Participants with an initial inadequate diet had an increase of $0.34 \mathrm{pp}(-0.06-0.74)$ after controlling for age, glycemic control, and 
Table 3 Mean change of the glycated hemoglobin (A1C) in percentage points (pp) by intial level of $A 1 C$ and standard deviation ( \pm SD) in patients of a Primary Health Care Service, according to the initial values and the association with covariables

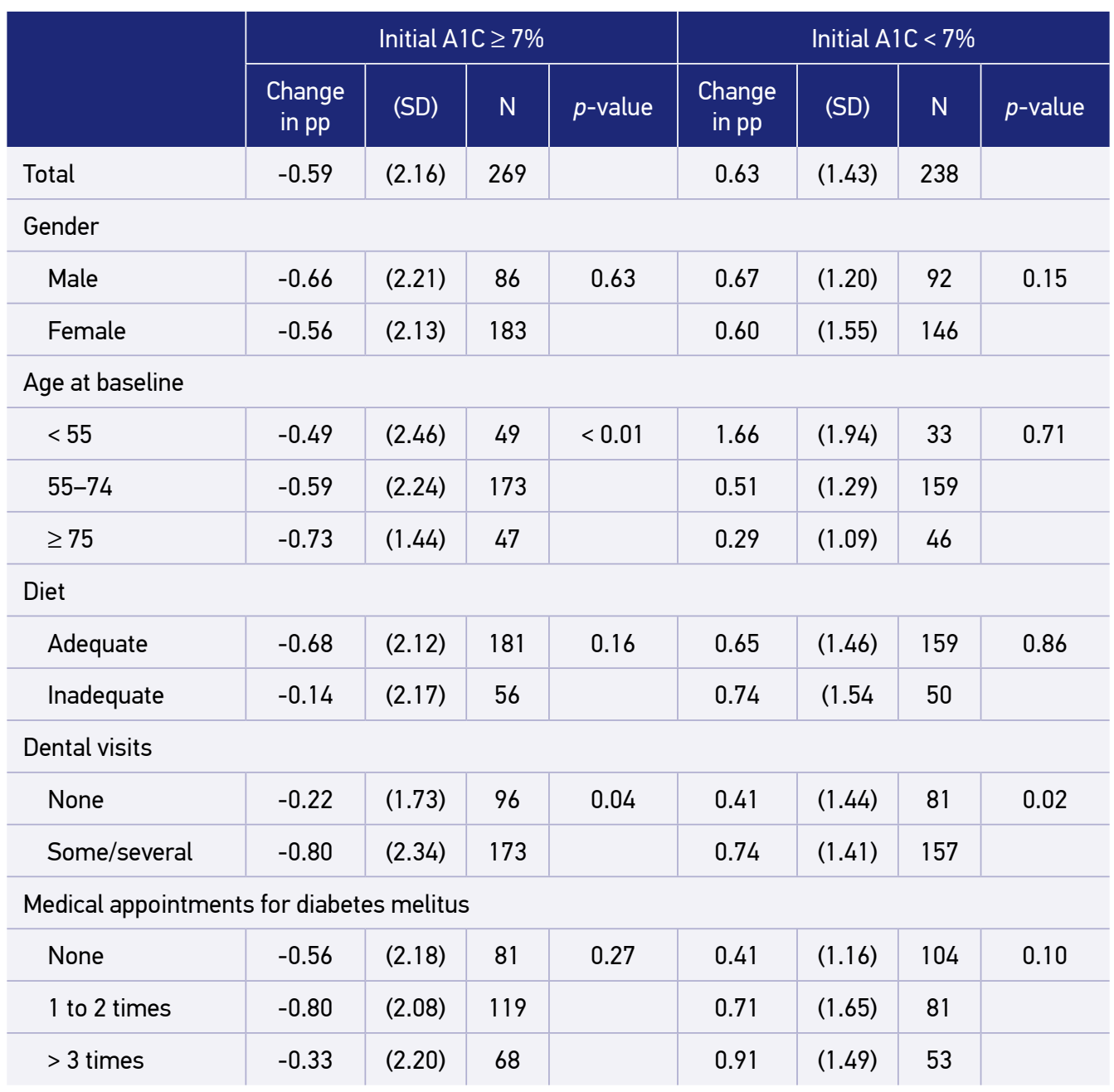

dental visits. As to dental visits, in an adjusted model, the well-controlled group presented a non-significant increase of $0.34 \mathrm{pp}(-0.18-0.87)$. Simultaneously, participants in the initial well-controlled group showed a reduction of $-0.56 \mathrm{pp}$ (95\%CI -1.06--0.07). When comparing the results of the two groups of patients (well-controlled versus not well-controlled), a statistically significant interaction was observed (coefficient for the interaction term 0.90 pp of A1C 95\%CI $1.63-0.18$; $\mathrm{p}=0.01$ ). The final model had an $\mathrm{R}^{2}=13.1 \%$, and the studentized residuals were normally distributed with some right-tail outliers that accounted for heteroskedasticity (Cook-Weisberg test for heteroskedasticity $\mathrm{p}<0.01$ ). Almost $10 \%$ of 
Table 4 Adjusted variation over the follow-up in glycated hemoglobin in percentage points ( $\mathrm{pp}$ ) and $95 \%$ confidence intervals $(95 \% \mathrm{Cl})$, obtained by multiple linear regression stratified by initial values

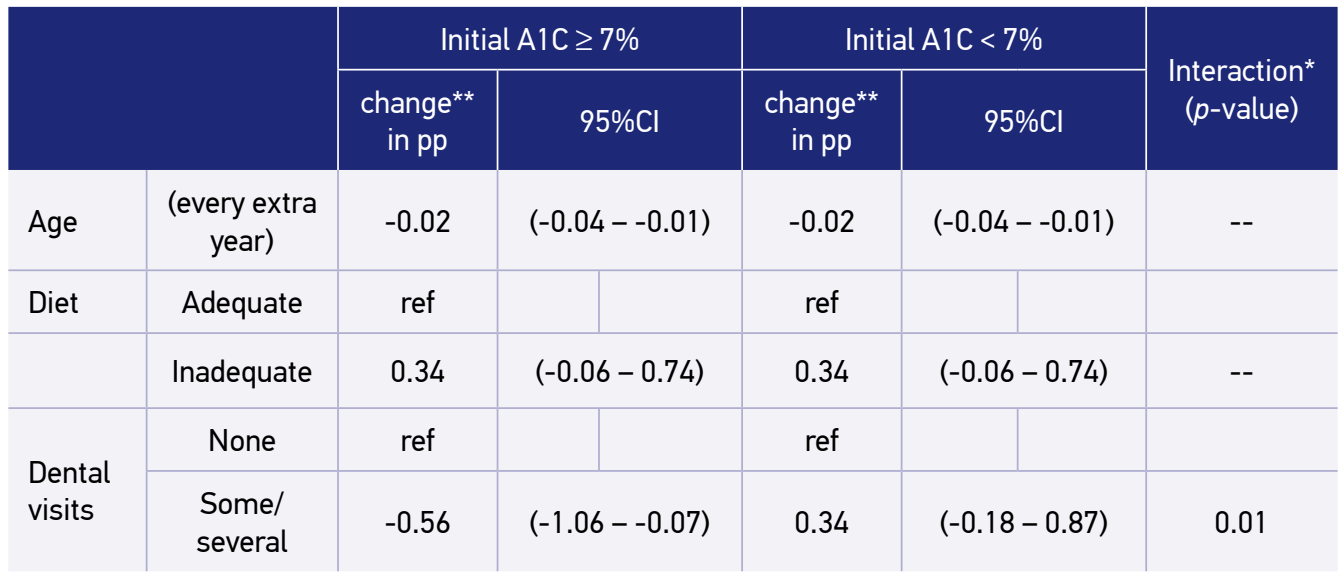

*This $p$-value refers to the interaction term (coefficient estimate $=0.90 \mathrm{~A} 1 \mathrm{C}$ p.p. $95 \% \mathrm{Cl} 1.63-0.18$ ) between the initial A1C and dental visits; ${ }^{* *}$ non-interaction terms are fixed coefficients (age and diet); they do not vary by the initial A1C categories.

the sample may be considered outliers. Concerning sensitivity analysis, analysis for outlier removal was reperformed, and the magnitude of interaction was only slightly stronger.

\section{DISCUSSION}

The present study showed that patients with initial A1C considered not well-controlled and who visited the dentist had an average reduction of approximately half percentage point in A1C. For example, from 7.56 to $7 \%$, that was statistically significant, whereas the group that already had a good glycemic control had a non-significant increase of $0.34 \mathrm{pp}$. Clinically, any improvement in glycemic control is likely to reduce the risks of diabetes complications and brings long-term benefits to patients. A previous study showed that each $1 \mathrm{pp}$ reduction in $\mathrm{A} 1 \mathrm{C}$ reduced the risk of any endpoint related to diabetes by $21 \%{ }^{5}$.

Different from our findings, a previous study did not find an association between dental visits (defined by the authors as having received prophylactic treatments or periodontal treatments) and glycemic control, choosing as an outcome the dichotomous measure A1C $<7 \%$ versus $\mathrm{A} 1 \mathrm{C}<7 \%$, not the continuous variation of $\mathrm{A} 1 \mathrm{C}$ as we $\mathrm{did}^{21}$. Another observational study ${ }^{22}$ found an association between dental visits related to periodontal treatment and glycemic level, and the reduction found for patients with an initial not well-controlled A1C was -0.035 pp lower than in the present study. The same authors did not find any association when the dental visits were not related to periodontal treatment. Nevertheless, only $8.2 \%$ of the sample with a diagnosis of T2DM had consulted the dentist for a non-periodontal reason, whereas $48.7 \%$ had received periodontal treatment. 
This study also showed that dental visits were associated (statistically non-significant) with an increase in $\mathrm{A} 1 \mathrm{C}$ in the controlled group. However, this may be a spurious finding, as there is no previous report to support it, and it does not seem plausible that dental visits would causally increase A1C levels. In support of this argument, CHS/ CHG introduced several management measures to improve the health indicators of patients with diabetes to increase both diagnosis and control. Examples include enhancement of access to dental care, an increase in the percentage of diagnosis, and the professionals' qualification involved ${ }^{18}$. Importantly, those measures were generic to all patients with DM and not specific to those well-controlled or not well-controlled. The analyses of present research sought to complement the understanding of the association of only one factor involved: expanding access to dental visits. However, other studies are necessary to measure the effectiveness of the other actions of this qualification.

Based on these managerial measures, there was an increase in the supply of dental visits in the public health service for patients with DM. In 2011, 16\% of all DM patients enrolled in the service had at least one dental visit, and it increased to $37 \%$ in $2016^{23}$. A study that qualitatively evaluated dental care in this health service found practices of a new health care model, characterized by clinical performance, beyond the traditional approaches, with more listening and dialogue with patients ${ }^{24}$. Nonetheless, dental care in CHS / CHG is integrated into general health, and dental teams are committed to working with effective prevention and health promotion, as shown elsewhere ${ }^{25}$. Therefore, all these efforts may help diabetic patients in controlling the disease.

The benefits of periodontal treatment for patients with T2DM are now widely known ${ }^{26,27}$. Although data on the dental condition were not available, people with not well-controlled T2DM had an increased prevalence of periodontitis and tooth $\operatorname{loss}^{9,14}$. Seen that, these patients could still have benefited more from dental treatment. The Study of Health in Pomerania (SHIP) - a trend that examined the periodontal status of 3,086 patients with T2DM and pre-diabetes found an association between periodontitis and edentulism with poorly controlled diabetes, but not in well-controlled and pre-diabetes patients. Besides that, Workers' Oral Health Study, which examined 5,154 participants in Spain, demonstrated that severe periodontitis was associated with diabetes ${ }^{8}$. Furthermore, the patients in the present study belonging to the not well-controlled group may have received increased attention from health teams concerning the need for blood glucose reduction, besides the benefit of greater control of a more severe periodontal condition.

Among the potentialities of present research, its longitudinal design, long follow-up period, large sample size, and insertion in a health service's practical scenario are highlighted. Besides that, few studies have been found separately evaluating patients with well and not well-controlled status.

Regarding study's limitations, residual confounding may exist, despite controlling for some important confounding measures available in the database, such as medication adherence, diet, BMI, socioeconomic conditions, schooling, marital status, attendance at diabetic and hypertensive groups, and perhaps changes may have occurred in this period. Additionally, no information was available about the type of dental treatment performed, whether it was 
completed or not, how it was performed, and for how long. It is also unknown if patients had sought dental treatment outside the CHS/ CHG services during the period and how it affected the results. Although some variables are self-reported, measurement bias and validity have been considered acceptable. For example, self-reported weight and height have a remarkably high intra-class correlation $(>0.94)$, and BMI has high sensitivity and specificity $^{28}$. Finally, the absence of data on dental conditions other than the number of teeth is a limitation that future studies should overcome.

With the increasing burden of morbidity and mortality, coping with T2DM has become a growing challenge in public health, and these patients may benefit from a common risk factor approach. An integrated approach to the main oral problems, such as diet, hygiene, smoking, alcohol use, stress, and trauma is more rational, increases effectiveness and efficiency, and reduces isolation ${ }^{17}$. This work is innovative, as it shows that the dental consultation for any reason and performed in Primary Health Care represent a positive impact on the $\mathrm{A} 1 \mathrm{C}$ reduction in a population with $\mathrm{T} 2 \mathrm{DM}$.

It also contributes to understanding the effects of a protocol aimed at increasing the number of dental visits among patients with T2DM. Encouraging visits to the dentist and promoting better oral health conditions may play a relevant role for patients with T2DM in PHC. Further studies are required to better understand all these factors and the relation between receiving dental visits and the A1C of patients with T2DM in PHC.

\section{REFERENCES}

1. NCD Risk Factor Collaboration. Worldwide trends in diabetes since 1980: a pooled analysis of 751 populationbased studies with 4.4 million participants. Lancet 2016; 387(10027): 1513-30. https:// doi.org/10.1016/ s0140-6736(16)00618-8

2. Bhutani J, Bhutani S. Worldwide burden of diabetes. Indian J Endocrinol Metab 2014; 18(6): 868-70. https: / / doi.org/10.4103/2230-8210.141388

3. Costa AF, Flor LS, Campos MR, Oliveira AF, Costa MFS, Silva RS, et al. Carga do diabetes mellitus tipo 2 no Brasil. Cad Saúde Pública 2017; 33(2): e00197915. https: / / doi.org/10.1590/0102-311x00197915

4. Duncan BB, França EB, Azeredo Passos VM, Cousin E, Ishitani LH, Malta DC, et al. The burden of diabetes and hyperglycemia in Brazil and its states: Findings from the Global Burden of Disease Study 2015. Rev Bras Epidemiol 2017; 20(Suppl. 1): 90-101. https:// doi.org/10.1590/1980-5497201700050008

5. Stratton IM, Adler AI, Neil HAW, Matthews DR, Manley SE, Cull CA, et al. Association of glycaemia with macrovascular and microvascular complications of type 2 diabetes (UKPDS 35): Prospective observational study. Br Med J 2000; 321(7258): 405-12. https: / / doi. org/10.1136/bmj.321.7258.405

6. D'Aiuto F, Gable D, Syed Z, Allen Y, Wanyonyi KL, White $S$, et al. Evidence summary: The relationship between oral diseases and diabetes. Br Dent J 2017; 222(12): 944-8. https: / / doi.org/10.1038/sj.bdj.2017.544

7. Graziani F, Gennai S, Solini A, Petrini M. A systematic review and meta-analysis of epidemiologic observational evidence on the effect of periodontitis on diabetes An update of the EFP-AAP review. J Clin Periodontol 2018; 45(2): 167-87. https: / / doi.org/10.1111/jcpe.12837

8. Montero E, Carasol M, Fernández-Meseguer A, Calvo-Bonacho E, García-Margallo MT, Sanz M, et al. Prediabetes and diabetes prevalence in the Workers' Oral Health Study. Clin Oral Investig 2019; 23(12): 4233-41. https: / / doi.org/10.1007/s00784-019-02875-3

9. Luo H, Pan W, Sloan F, Feinglos M, Wu B. Fortyyear trends in tooth loss among american adults with and without diabetes mellitus: An age-period-cohort analysis. Prev Chronic Dis 2015; 12(12): E211. https: / / doi.org/10.5888/pcd12.150309 
10. Latti BR, Kalburge JV, Birajdar SB, Latti RG. Evaluation of relationship between dental caries, diabetes mellitus and oral microbiota in diabetics. J Oral Maxillofac Pathol 2018; 22(2): 282-3. https://doi.org/10.4103/ jomfp.jomfp_163_16

11. Hintao J, Teanpaisan R, Chongsuvivatwong V, Dahlen G, Rattarasarn C. Root surface and coronal caries in adults with type 2 diabetes mellitus. Community Dent Oral Epidemiol 2007; 35(4): 302-9. https: / / doi. org/10.1111/j.1600-0528.2007.00325.x

12. Yamashita JM, Moura-Grec PG, Capelari MM, SalesPeres A, Sales-Peres SHC. Manifestações bucais em pacientes portadores de Diabetes Mellitus: uma revisão sistemática. Rev Odontol UNESP 2013; 42(3): 211-20. https: / / doi.org/10.1590/S1807-25772013000300011

13. Preshaw PM, Alba AL, Herrera D, Jepsen S, Konstantinidis A, Makrilakis K, et al. Periodontitis and diabetes: A two-way relationship. Diabetologia 2012; 55(1): 21-31. https: / / dx.doi.org/10.1007\%2Fs00125-011-2342-y

14. Löe H. Periodontal disease: The sixth complication of diabetes mellitus. Diabetes Care 1993; 16(1): 329-34.

15. Miranda TS, Heluy SL, Cruz DF, Silva HDP, Feres M, Figueiredo LC, et al. The ratios of pro-inflammatory to anti-inflammatory cytokines in the serum of chronic periodontitis patients with and without type 2 diabetes and/ or smoking habit. Clin Oral Investig 2019; 23(2): 641-50. https: / / doi.org/10.1007/s00784-018-2471-5

16. Genco RJ, Genco FD. Common risk factors in the management of periodontal and associated systemic diseases: The dental setting and interprofessional collaboration. J Evid Based Dent Pract 2014; 14(Suppl.): 4-16. https:// doi.org/10.1016/j.jebdp.2014.03.003

17. Sheiham A, Watt RG. The common risk factor approach: a rational basis for promoting oral health. Community Dent Oral Epidemiol 2000; 28(6): 399-406. https:// doi.org/10.1034/j.1600-0528.2000.028006399.x

18. Baldisserotto J, Kopittke L, Nedel FB, Takeda SP, Mendonça CS, Sirena SA, et al. Socio-demographic caracteristics and prevalence of risk factors in a hypertensive and diabetics population: A cross-sectional study in primary health care in Brazil. BMC Public Health 2016; 16: 573. https:// doi.org/10.1186/s12889-016-3230-7

19. De Lima LA, Nedel FB, Olinto MTA, Baldisserotto J. Food habits of hypertensive and diabetics cared for in a primary health care service in the South of Brazil. Rev Nutr 2015; 28(2): 197-206. https: / / doi. org/10.1590/1415-52732015000200008

20. World Health Organization. Obesity: preventing and managing the global epidemic. Report of a WHO consultation. Geneva: World Health Organization; 2000. Technical Report Series, v. 894.
21. Mosen DM, Pihlstrom DJ, Snyder JJ, Shuster E. Assessing the association between receipt of dental care, diabetes control measures and health care utilization. J Am Dent Assoc 2012; 143(1): 20-30. https: / / doi.org/10.14219/ jada.archive.2012.0014

22. Saito M, Shimazaki Y, Nonoyama T, Tadokoro Y. Association between dental visits for periodontal treatment and type 2 diabetes mellitus in an elderly Japanese cohort. J Clin Periodontol 2017; 44(11): 11339. https:// doi.org/10.1111/jcpe.12804

23. Brasil. Ministério da Saúde. Grupo Hospitalar Conceição. Indicadores de Saúde do SSC. Informe Mensal. Brasil: Ministério da Saúde; 2017. 127 p.

24. Graff VA, Toassi RFC. Oral health clinic as a space for the production dialogue, connection and subjectivity among users and dentists of primary care. Physis 2018; 28(3): e280313. https:// doi.org/10.1590/ s0103-73312018280313

25. Celeste RK, Nadanovsky P, De Leon AP. Association between preventive care provided in public dental services and caries prevalence. Rev Saude Publica 2007; 41(5): 830-8. https://doi.org/10.1590/ S0034-89102007000500018

26. Simpson TC, Weldon JC, Worthington HV, Needleman I, Wild SH, Moles DR, et al. Treatment of periodontal disease for glycaemic control in people with diabetes mellitus. Cochrane Database Syst Rev 2015; 2015(11): CD004714. https:// doi.org/10.1002/14651858. cd004714.pub3

27. Madianos PN, Koromantzos PA. An update of the evidence on the potential impact of periodontal therapy on diabetes outcomes. J Clin Periodontol 2018; 45(2): 188-95. https: / / doi.org/10.1111/jcpe. 12836

28. Fonseca MJM, Faerstein E, Chor D, Lopes CS. Validity of self-reported weight and height and the body mass index within the "Pró-saúde" study. Rev Saude Publica 2004; 38(3): 392-8. https: / / doi.org/10.1590/ S0034-89102004000300009

Received on: 01/06/2021

Revised on: 03/01/2021

Accepted on: 03/17/2021

Authors' contribution: ALH originated and designed the study, and drafted the manuscript. JB originated and designed the study, acquired data, interpreted data, and critically revised the manuscript for important intellectual content. RKC originated and designed the study, analyzed and interpreted data, and critically revised the manuscript for important intellectual content. 